\title{
LA TEORÍA DE ERNST TUGENDHAT SOBRE LAS FUENTES DE LA MORAL
}

\author{
ERNST TugENDHAT ON THE SOURCES OF MORALITY
}

\author{
Ursula Wolf
}

\begin{abstract}
RESUMEN
Para Tugendhat, el principal motivo moral de su enfoque contractualista es el deseo de ser reconocido como miembro de la comunidad contractual. Entre otras cosas, este motivo no puede justificar la prohibición de la crueldad con los animales. Con respecto a este problema, Tugendhat fluctúa entre la adopción de un segundo motivo, la compasión, y la externalización de la actitud hacia los animales y la naturaleza en su conjunto al misticismo. El presente artículo intenta resolver este problema explicando la relación humano-animal y elaborando sus implicaciones éticas. Se argumenta que la moralidad se refiere generalmente a la consideración del bienestar de los seres que son capaces de sufrir. Puesto que el bienestar de estos individuos tiene diferentes dimensiones y nuestras relaciones con ellos también tienen diferentes tipos, de la misma manera la acción moral se basa en diferentes motivos, sin que tengamos que asumir una tensión entre motivos incompatibles.
\end{abstract}

PALABRAS CLAVE: Moralidad, contractualismo, motivación moral, animales, naturaleza, bienestar, compasión, misticismo.

\section{ABSTRACT}

The main moral motive for Tugendhat's contractualist approach is the desire to be recognized as a member of the contractual community. But this reason can not justify the prohibition of cruelty to animals. With regard to this problem, Tugendhat fluctuates between the adoption of a second motive, compassion, and the externalization of the attitude toward animals and nature as a whole to mysticism. This article attempts to solve this problem by explaining the human-animal relationship and its ethical implications. It is argued that morality generally refers to the consideration of the welfare of beings who are capable of suffering. Since the welfare of these individuals has different dimensions and our relationships with them also have different types, so the moral action is based on different reasons, without having to assume a tension between incompatible motives.

KEYWORDS: Morality, contractualism, moral motivation, animals, nature, welfare, compasion, mysticism. 
Como ocurre con todos los grandes filósofos, encontramos también en la filosofía de Ernst Tugendhat determinados puntos clave sobre los que él gira durante toda su vida. En la filosofía moral es la idea de que el núcleo de la moral se encuentra en una concepción mínima, la cual se construye de modo contractualista y se justifica en el propio interés. Lo que el contractualismo provee es el contenido de las normas básicas. Sin embargo, de esta primera pregunta de justificación (la del contenido), Tugendhat diferencia una segunda, la de la motivación, por qué nos comprometemos con la moral y la cumplimos. ${ }^{1}$ Esta segunda pregunta desempeña un papel importante en la teoría moral de Tugendhat, ya que para él no se trata de que los individuos obedezcan una moral ciegamente, sino de que se sometan a ella de forma autónoma. El motivo para obedecer que él cita excede la mera utilidad del actor; consiste en el deseo de ser respetado como miembro en la comunidad contractual o de evitar la sanción (informal, no jurídica) del desprecio por parte de los otros. También este deseo se clasifica naturalmente en el propio interés en un sentido amplio.

Pero como el mismo Tugendhat a veces admite, esta motivación no es suficiente para acomodar todo lo que incluimos en la moral en la vida cotidiana. Por ejemplo, de esta forma no puede concebirse la prohibición del maltrato a animales, pues los animales no son partes contratantes que nos puedan sancionar por menosprecio si los perjudicamos. Pero entonces se cuestiona si, además del interés propio, tenemos que presuponer una segunda fuente motivacional de la moral. De hecho, Tugendhat, en una versión más tardía de su filosofía moral ("Das Problem einer aufgeklärten Moral", 2005), adopta dos fuentes de una moral autónoma, el interés propio y la compasión natural, un altruismo sentimental (23). La compasión fomenta, como él dice, el actuar moralmente, y de ahí avanza, en cuanto que se convierte en una actitud generalizada, en una virtud moral. De esta forma, en la moral contractualista entra un elemento que sobrepasa esta moral en cuanto al alcance de su contenido. Mientras que la moral contractualista se limita al grupo de los que se pueden obligar mutualmente, la actitud generalizada de compasión se extiende a todos los seres con capacidad de sufrimiento, o sea también a los animales.

¿Cómo se ajusta esta duplicación del fundamento motivacional de la moral al simple enfoque contractualista? El hecho de que exista aquí un problema es constatado por el propio Tugendhat en varios de sus escritos. En la temprana monografía Vorlesungen über Ethik (1993, 189-192), Tugendhat separa el altruismo de la moral que se extiende hacia los animales. Bien es verdad que, en ella, al igual

1 Véase, por ejemplo, en Tugendhat 1993, 29, 85. 
que en el artículo más posterior, admite que la crueldad es un vicio que tiene que ser condenado moralmente, pero entonces piensa que es necesaria una nueva aclaración de la relación hombre-animal. En el escrito posterior dice que la existencia de dos fuentes diferentes de la moral implica una tensión indisoluble dentro de la conciencia moral. Sin embargo, al final del texto no está del todo claro si él deja persistir la tensión. Parece que también aquí quiere apartar el altruismo de la moral. Pero, al mismo tiempo, el altruismo va a ser revalorizado. Pues la solución sobre cómo se ha de clasificar al altruismo consiste en entender la benevolencia generalizada no como virtud moral sino como una actitud más profunda, o sea como una actitud mística (24).

Pero antes de buscar refugio en la mística pienso que se debería tomar en serio la exigencia en el ensayo anterior, es decir, intentar explicar la relación hombreanimal más detalladamente. Pues solo así se puede averiguar cuáles son los aspectos morales implicados en esta relación y cuál es su naturaleza. Esto es lo primero que deseo hacer a continuación. ${ }^{2}$ En un segundo paso voy a sacar conclusiones en cuanto al contenido de la moral. En un tercer paso me ocupo de la forma de la moral ilustrada desde una cierta comprensión de esta forma. En cuarto lugar, pregunto por las fuentes de la moral, para finalmente formular en quinto lugar una respuesta a la alternativa de Tugendhat entre moral contractualista y compasión. ${ }^{3}$

A continuación, trabajo con una diferencia entre tres aspectos de la moral: forma, motivo y contenido. Con "forma" me refiero a lo que intenta Tugendhat con el concepto general de una moral; con "contenido" denomino la totalidad de las normas o virtudes, lo cual constituye un punto de vista moral determinado; con "motivo" me refiero a los estímulos psicológicos que nos inducen a la acción moral. Además, al igual que Tugendhat, hablo de las "fuentes de la moral" y empleo este término sin una fijación estricta; se refiere tanto a motivos como a lo que pertenece a la forma de la moral (de hecho, la forma y el motivo a veces no pueden separarse).

\section{Aspectos morales de la RELACión hombre-ANimal}

Si seguimos a Tugendhat, tenemos a día de hoy dos concepciones morales diferentes: un punto de vista de consideración universal, igual y recíproca que vale

\footnotetext{
2 He explicado estas relaciones más detalladamente en Wolf 2012, cap. III. Una diversidad de relaciones, o sea, fuentes de la moral, es propuesta, entre otros, por Midgley 1993, Scruton 1996 y Warren 1997.

${ }^{3}$ Las consideraciones siguientes se basan parcialmente en mi artículo Wolf 2005.
} 
para personas capaces de razonar, y un punto de vista del altruismo, de la compasión generalizada, que comprende la extensión de la moral más ampliamente al incluir como objetos a todos los seres con capacidad de sufrimiento. Para Tugendhat, el primero de estos puntos de vista es el central, ya que su propio interés se dirige a la justificación autónoma de la moral. Ahora quiero avanzar a la inversa, o sea ver, en primer lugar, qué fenómenos variados incluimos en la moral, y después buscar una teoría moral que los pueda acomodar a todos ellos. Para eso voy a examinar el caso de los animales, el cual es difícil de concebir en todas las morales racionalistas.

Parto de que hoy en día está generalmente aceptado que no se debe maltratar a animales arbitrariamente, que se exigen determinadas consideraciones morales también hacia ellos. Si examinamos las distintas relaciones con animales, enseguida se observa que el modelo bipartito de Tugendhat no es suficiente. Así, en lo que concierne a los animales útiles que viven dentro de la comunidad humana, el punto de vista de la compasión seguro que no basta. Por supuesto que juega también un papel —no deberíamos maltratar a animales cuando ya los utilizamos, o sea no deberíamos mantenerlos bajo condiciones en las que sufran dolor, miedo o estrés, o en lugares en los que les falta movimiento, trato con congéneres, etc- - Pero lo importante - y esto es válido para todos los animales domésticos, también para las mascotas - es algo diferente: al haber (nosotros) domesticado a animales, al haberlos sacado de su entorno natural y al haberlos privado de este modo de la posibilidad de cuidarse de sí mismos, estamos obligados a asegurar su sustento. Bien es verdad que puede decirse que hay razones instrumentalistas para este cuidado, al querer utilidad de parte de los animales. Pero para esto no se necesita cuidar a cada animal uno por uno, y tampoco cuidarlos considerando el modo de su vida natural. Eso significa que la exigencia moral no sobra. Hay dos posibilidades de concebirla:

Primero, es obligatorio cuidar de seres que (independientemente de las razones) se hallan bajo nuestra custodia y son dependientes, es decir, no son capaces de cuidarse de sí mismos; por ejemplo, este es el caso de los niños pequeños. ${ }^{4} \operatorname{Sin}$ embargo, los animales útiles no son seres dependientes en este sentido, sino que nosotros los hemos puesto en una situación en la que ya no tienen la posibilidad de cuidarse de sí mismos. Entonces, en segundo lugar, existiría la posibilidad de hablar aquí de una relación cuasicontractual y decir que nosotros cuidamos de sus condiciones de vida como contrapartida por la utilidad que nos aportan (la diferencia con respecto al contrato consiste naturalmente en que los animales no

\footnotetext{
${ }^{4}$ MacIntyre 1999 apunta la existencia de tales obligaciones unilaterales de cuidado en la moral entre humanos.
} 
han decidido la cooperación por sí mismos). Pero básicamente basta la obligación del cuidado, pues esta se mantendría incluso en situaciones en las que un animal ya no aporta una utilidad.

Algo cuasicontractual podría verse en el caso especial de las mascotas. Animales altamente desarrollados como los perros o los gatos pueden establecer una relación estrecha en la convivencia e interacción con las personas. Tales relaciones generan expectativas y, en consecuencia, obligaciones correspondientes.

La actitud de la compasión generalizada parece más bien adecuada en aquellos casos en los que se trata del comportamiento hacia animales que viven en libertad. Cuando las personas cazan, apresan o utilizan de algún modo a los animales en libertad, podría decirse que se tiene que evitar exponerlos al sufrimiento. Pero también en lo relacionado con los animales que viven en libertad, la compasión no es siempre la actitud moral adecuada. Por ejemplo, existen frecuentemente conflictos de interés entre persona y animal que conciernen la alimentación o el espacio. En un conflicto de interés entre personas no se trata de una cuestión de la compasión, sino de una solución justa que exige que todos restrinjan sus intereses para poder así satisfacer en paz sus intereses restantes.

El problema es que de los animales no podemos esperar una restricción voluntaria de intereses. Si alguien quiere construir una casa en un terreno hasta ahora salvaje, con los animales que allí viven no puede negociar una solución que sea aceptable para ambas partes. Pero, de todas formas, en cuanto conozcamos las necesidades de los animales afectados por una medida humana, podemos tenerlas en cuenta. En aquellos casos en los que, según nuestro cálculo, se puede encontrar una regulación que es aceptable para ambas partes, no nos queda otro remedio que imponérsela a los animales; pero eso, además de la limitación de intereses, puede originar sufrimiento adicional, lo que habría de incluirse en el cálculo como un factor adicional. Pero quizás podría decirse que ese sufrimiento es insignificante en los casos en los que tiene una medida soportable y solo surge a corto plazo.

Pero si se reflexiona sobre esta constelación hasta el final, chocamos rápidamente con un límite de la posibilidad de una compensación justa de intereses. La humanidad se ha extendido tanto por la Tierra que cada vez hay menos espacio y medios de subsistencia para los otros animales, lo que puede causar hambre, lesiones, miedo o estrés. También aquí se puede decir, por supuesto, que cada persona ha de esforzarse para que los animales que viven en su entorno sufran lo menos posible, y que cada persona ha de ayudar a animales necesitados con los que se vea confrontado. Pero las repercusiones del modo de vida humano en los animales son frecuentemente indirectas y no siempre eludibles, y considerar a los animales 
como seres con capacidad de sufrimiento choca por eso con el límite de lo practicable. Aquí solo queda sentir lástima de que el modo de vida humano tenga estos efectos en el mundo animal. Y en este punto se podría, quizás, dar un paso a la mística, o sea considerar, si así se quiere, esta comprensión de la inalcanzabilidad de una práctica moral universal como una actitud mística.

\section{LA DIVERSIDAD DE LOS CAMPOS DE LA MORAL}

En las relaciones entre persona y animal se muestra, pues, una multitud de aspectos morales: el principio de no infligir sufrimiento y ayudar en emergencias; el deber de cuidado para con seres dependientes que hemos acogido o, si se trata de menores humanos, que hemos adoptado o engendrado; la exigencia de una regulación justa en conflictos de intereses; el cumplimiento de expectativas que se originan en la vida común y acciones cooperativas. Entre las personas adultas se añade el respeto y la consideración mutua, y el cumplimiento de contratos. Desde el principio no parece prometedor encontrar una fuente única para estos contenidos. Pues, para esto, los principios nombrados tendrían que reducirse a un principio solo fundamental. ¿Pero qué principio podría ser este? Según Tugendhat, el de una consideración igual recíproca. Pero en esto no se puede concebir nuestra relación con los animales. Por otro lado, al borrar la palabra "recíproco" y mencionar solo la consideración universal, se acentúan las obligaciones negativas y no se incluyen las obligaciones del cuidado. En lugar de esto, ¿podría decirse, partiendo de la compasión, que el principio básico consiste en tratar a otros seres como seres con capacidad de sufrimiento? Pero como hemos visto, eso no es apto en los casos en que la cuestión es la solución justa de conflictos. Y tampoco el cuidado de un ser dependiente es asunto de la compasión, sino una obligación que se origina en una constelación especial.

Si es que hay en realidad un aspecto común para estos temas diversos de la moral, este no se encuentra en un principio de actuación unitario sino, en el mejor de los casos, en la propiedad que todos los seres afectados tienen en común. Ya que se incluye a los animales, es natural ver esta propiedad en la capacidad de sufrimiento o de sentimiento. Pero esto, expresado de este modo, podría dar lugar a malentendidos como los que le ocurren al utilitarismo, o sea a contemplar aisladamente las sensaciones de placer y de displacer, de modo que los individuos jueguen un papel solo como vasos de tales sentimientos. ${ }^{5}$ La descripción de la

5 Este es el reproche que Regan hace al utilitarismo en el debate de la ética animal, compárese con Regan 1984, 205ss. 
compasión de Schopenhauer nos lleva adelante en este punto. Como él dice, en la compasión nos referimos al bienestar y malestar de otro ser. Por eso son objeto de la moral seres que tienen un bienestar y malestar, es decir, que realizan una vida de modo que les pueda ir mejor o peor subjetivamente. Entonces los distintos campos morales y sus fuentes podrían recuperarse de tal manera que uno se pregunta: cuáles son los ámbitos fundamentales del bienestar subjetivo; qué interrelaciones son en principio posibles entre el bienestar de seres individuales diferentes; qué consecuencias puede tener la actuación común de muchos sobre el bienestar de los individuos.

\section{LA DERIVACIÓN DE LA MORAL ILUSTRADA}

En contra de determinar la extensión de la moral siguiendo a Schopenhauer, Ernst Tugendhat objetaría que eso no conduce a una moral, ya que en esta descripción falta la referencia a las normas que llevan sanciones. Una moral, como él dice, es esencialmente un sistema de tales normas vinculadas a sanciones internalizadas que consisten en los afectos morales de la vergüenza y la culpa en la persona que incumple una norma y, respectivamente, en la indignación cuando se observa cómo se maltrata a otros. Pues bien, hasta ahora solo he nombrado una cualidad que podría convertir a los seres en objetos de la moral. Por el contrario, acabo de poner en duda que tiene sentido formular como contenido de la moral la exigencia general de considerar el bienestar subjetivo de todos o actuar en el sentido de una compasión generalizada. Hasta el momento apenas he hablado sobre qué normas resultan de las dimensiones de esta cualidad y de los tipos de interrelaciones entre seres con estas cualidades. Cualesquiera que sean estas normas, el hecho de que se deriven de las dimensiones del bienestar seguramente no impide aceptar por principio un concepto moral general como el que propone Tugendhat. Aunque Schopenhauer no lo haga, esto es posible y es defendido por la otra teoría ilustrada con igual alcance que la moral de la compasión, es decir, el utilitarismo.

Así, Mill, en el tercer capítulo de su libro Utilitarismo, explica que para cada punto de vista moral surgen las preguntas de con qué sanción está conectado, por qué motivo se obedece y de dónde proviene su fuerza vinculante u obligatoria. Según Mill, estas preguntas no confrontan especialmente el principio de utilidad, más bien el utilitarismo puede recurrir a aquellas respuestas que son válidas para cada punto de vista moral. Al mismo tiempo, Mill es más detallado que Tugendhat en la descripción del marco general de cada moral. Mill cita sanciones externas e internas de tipo social y religioso (entre otras, favor o enojo en los prójimos, 
sentimientos de culpa, autoestima y apreciación externa, conciencia); también cita afectos altruistas relacionados con el individuo como amor y simpatía; y sentimientos relacionados con la sociedad en general, como el sentimiento de comunidad. Mill considera en principio posible el que un individuo pueda cuestionar estas estructuras y pueda intentar deshacerse de ellas, pero no lo considera como una opción real contra la que se necesite argumentar. No lo considera sobre todo porque Mill — como la ética antigua — otorga mucha importancia a la eficacia de la educación: tan pronto como nos preguntamos autónomamente por el punto de vista moral correcto, estamos ya siempre socializados y nos hallamos situados en una red de dependencias sociales ${ }^{6}$. Y, según Mill, esta socialización está tan profundamente anclada en la persona que se la incluye usualmente casi dentro de nuestras cualidades naturales.

Si se toma por base tal punto de vista diferenciado del concepto de la moral, o sea de la forma y del motivo de la moral, entonces el esfuerzo de Tugendhat por la derivación de la moral ilustrada parece muy distante de la realidad, y la contradicción entre el altruismo y la autonomía parece artificial. Por supuesto, puede ser que él no considere la derivación como realista. Pero también en cuanto a los conceptos apenas es posible derivar un motivo social a partir de uno egoísta. La explicación de Tugendhat se refiere a la situación en la que un niño pregunta por un motivo de la conducta moral. Ahora la situación de un cuestionamiento radical y de un esfuerzo por aspirar a la autonomía no es la del niño sino la del adolescente en pubertad. El último ya está socializado, pero quiere desligarse del mundo de los adultos y ganar independencia, y para esto solo queda rechazar aquello que los adultos exigen. Pero esta apenas es una situación que cuestiona fundamentalmente las normas morales. Pues se puede observar que los jóvenes sí muestran solidaridad y consideración dentro de su grupo siempre que hayan crecido en condiciones no totalmente antisociales. El hecho de que de forma pasajera no se comportan con respeto frente a personas mayores a quienes realmente deben agradecimiento apenas puede cambiarse por la referencia al propio interés en un sistema de normas recíproco. Presumiblemente este comportamiento va a cambiar a medida que los adolescentes vayan construyendo sus propias estructuras vitales y tomen responsabilidad dentro de ellas. Así, desarrollan una comprensión de lo que significa ser una persona independiente y, simultáneamente, figurar en variadas redes e interdependencias sociales.

6 También lo critica MacIntyre, op. cit., 82. 
En este contexto tener autonomía puede solo significar el no aceptar obligaciones que otros o la sociedad presentan casualmente, sino comprobar por sí mismo qué obligaciones tienen un fundamento y cuáles no lo tienen. El concepto de autonomía de Tugendhat no me resulta totalmente comprensible porque él ve una justificación autónoma solo cuando se refiere a motivos egoístas. O bien resuenan aquí los restos de un existencialismo individualista, o bien se trata de un intento de separar el concepto kantiano de la autonomía del concepto metafísico de razón y transmitirlo a la razón instrumental. El recurrir a motivos egoístas no puede explicar el significado de la actuación autónoma porque la cuestión de la autonomía se plantea igualmente para los motivos egoístas. También estos están marcados por la socialización y, si se reflexiona críticamente sobre su origen y su contenido, podrían mostrarse motivos de tal tipo que uno no los quiera integrar en su imagen propia.

\section{FuENTES DE LA MORAL}

Si enlazamos con la descripción de Mill del concepto general de moral y tenemos en cuenta la diferenciación previamente hecha de diversos contextos morales, se disuelve también la presunta tensión entre el contractualismo y la compasión. Como he explicado, Mill cita varias raíces de la moral: simpatía, amor, afectos morales, educación, sentimiento de comunidad, etc. Las relaciones ya variadas en el comportamiento con los animales indican igualmente diferentes bases de la moral. En el comportamiento con animales que viven dentro de la sociedad humana, la fuente es el cuidado, para lo cual el paradigma en el campo humano es el amor paternal, y con las mascotas, más allá, podría ser un amor afectivo o la amistad. En cuanto a los animales de compañía también juega un papel la reciprocidad, que crea esperanzas y obligaciones.

Más obvia es la génesis de obligaciones en la interacción entre personas. Consideramos ahora el uso de servicios humanos. En un entorno de libertad tal interacción se realiza mediante acuerdos que crean un contexto institucional que define, por las dos caras, tanto obligaciones como derechos. De esta manera también prometer algo es una acción que produce un hecho institucional, es decir, cuyo sentido es generar una obligación. ${ }^{7}$ En tales acciones institucionales encontramos una fuente inofensiva para el concepto de obligación que no necesita más explicación. De forma similar, se podrían explicar las expectativas recíprocas que se origi-

\footnotetext{
7 Lo indica Searle en Searle 1969, cap. 8.
} 
nan en las relaciones personales, o sea mediante el intercambio efectivo de muestras de amistad entre personas. De verdad hay razones para limitar el discurso de obligación moral y los afectos específicos de indignación, rencor o sentimiento de culpa a este campo de las obligaciones especiales; pues aquí estos fenómenos tienen un sentido no misterioso como implicaciones de acciones que producen obligaciones por su propio sentido. ${ }^{8}$ Sin embargo, esta fuente de la moral, la cual se muestra de parte de las personas en la fiabilidad moral, se extiende solo tan lejos como los entrelazamientos interpersonales concretos.

Aunque los conflictos de interés también tienen que ver con la interacción, en ellos no se trata de cumplir obligaciones especiales, sino de encontrar estructuras justas. Estas pueden concernir bien a la prevención de molestias mutuas o bien a la asignación de bienes. Aquí, de hecho, el contractualismo parece ser el modelo más apropiado. Sin embargo, las cuestiones de distribución no son exactamente cuestiones del individuo sino de la moral política, para la cual realmente quizás no disponemos de más que de la concepción contractual.

En los casos en los que se trata de arreglos entre individuos, la fuente del acuerdo moral podría verse en un interés por vivir en una comunidad bien formada, en la cual los individuos se reconozcan recíprocamente como tales que llevan una vida con necesidades e intereses. En lo que incumbe a encontrar un acuerdo, aquí no se trata de fiabilidad moral de las personas, como ocurre con las obligaciones especiales, sino de una actitud de equidad o de la consideración de las necesidades de otros. De quien no posee esta actitud podría decirse que no tiene sentido para el interés general, que es egocéntrico, ambicioso o codicioso. Después de haber llegado a un arreglo, la fuente para su cumplimiento es, otra vez, aquella que resulta de las obligaciones especiales. Tanto obligaciones especiales como exigencias de equidad adquieren su sentido de estructuras limitadas, las primeras provenientes de relaciones especiales, las segundas de la relación con un estado determinado.

Contemplamos ahora tal comportamiento moral que se mostró exigible frente a los animales salvajes, es decir, la norma de no infligir sufrimiento a ningún otro y de ayudar en caso de emergencia. Este parece ser el campo de la moral con la extensión más amplia. Es independiente de la existencia de relaciones de interacción efectivas, pero también del interés por establecer regulaciones comunes. Abarca cualquier ser con el que se tome contacto. Aquí, la fuente consiste en la

8 Como Williams dice en Williams 1985, 180, hablamos solo aquí de obligación en un sentido corriente, mientras que la ampliación del concepto de obligación a todos los campos de la moral causa problemas. 
capacidad de compasión. También los afectos con los que reaccionamos a la ausencia de esta capacidad son diferentes al caso de las relaciones interactivas. Suena extraño decir que, por ejemplo, un sádico que tortura a animales (y esto también es válido en el caso de las personas) nos provoca indignación. La reacción adecuada aquí es, más bien, el espanto o el asco. Correspondientemente, el delito moral no parece aquí como el incumplimiento de un deber, sino como la manifestación de barbarie y crueldad.

Todos los fenómenos nombrados tienen en común algún tipo de relación con el bienestar o la vida buena individual o común. Pero esta nota general no conduce a proposiciones sustanciales sobre la moral. Estas no son alcanzadas antes de que aclaremos las diversas relaciones entre seres, las estructuras de acción, los afectos o las actitudes que constituyen las diferentes partes de la moral. A este respecto solo he dado algunas indicaciones aproximativas que habría que desmenuzar algún día.

\section{LA TESIS DE LA CONTRADICCIÓN ENTRE LA MORAL RACIONAL Y LA COMPASIÓN}

De las consideraciones expuestas hasta ahora resulta que la tensión que Tugendhat supone entre la moral racional, o sea la moral justificada de modo egoísta y la moral de la compasión, apenas corresponde a la complejidad de los fenómenos morales. En primer lugar, no es posible derivar del egoísmo un motivo de actuar moralmente. En lugar de esto, habría de decirse: hay más fuentes de la moral además de la compasión, y la compasión también existe como fuente. Sin referencia a la compasión y a las otras fuentes de la moral mencionadas a nivel de los afectos ordinarios no se puede comprender cómo el actuar moralmente es posible. Y es esto y nada más lo que Schopenhauer quiere mostrar cuando se refiere a la compasión.

Para todas estas fuentes es válido, como Mill señala, que se han transformado en parte de nuestra naturaleza en cierto sentido. $\mathrm{O}$, hablando con Aristóteles: se han transformado en disposiciones fijas del carácter que contienen afectos al igual que apetencias con las que reaccionamos inmediatamente a las situaciones de acción relevantes. Ahora bien, Aristóteles ya ve que una virtud está ciega sin deliberación. Incluso quien solo posee buenas cualidades de carácter puede actuar con equivocaciones morales en una situación concreta si él no se da cuenta de las necesidades de tal situación mediante una deliberación práctica. Por eso, Tugendhat tiene razón en que la mera compasión puede llevar también a la equivocación. Esto mismo también es válido en el cumplimiento ciego del deber, del cuidado o del amor. 
No obstante, en todos estos campos es válido lo que Tugendhat menciona como la intuición por detrás de la moral de la compasión: si alguien actúa casi espontáneamente en el sentido de una de las fuentes afectivas de la moral, esto nos parece mejor que si lo hace solo porque lo considera moralmente correcto por un juicio. Este no es el punto de Schopenhauer, pues él mismo es de la opinión de que la compasión, que en realidad está limitada, debería consolidarse como una disposición general permanente. Más bien este es el punto de Schiller contra la posición de Kant, como él la entiende, de que la idea antigua de una armonía entre reacción afectiva y deliberación es más convincente que la concepción de Kant diciendo que una acción es tanto más valiosa cuanto más vaya en contra de nuestra inclinación.

Aunque la concepción antigua parece más adecuada a los fenómenos que la concepción de Kant, se mantienen dos problemas: primero, que puede faltar la base afectiva de una acción moral. Segundo, que pueden presentarse situaciones en las que entren en conflicto dos de tales motivos morales en el nivel inferior de los afectos ordinarios. La primera posibilidad se da cuando, en un caso determinado, alguien a causa de deseos propios fuertes no actúa conforme a la actitud de la compasión generalizada. La segunda posibilidad podría consistir en que, por ejemplo, compasión y equidad generen exigencias que el actor no puede cumplir al mismo tiempo. Esta posibilidad muestra que nosotros, en principio, hemos de remitirnos siempre al nivel de la deliberación, a la apreciación ponderadora de la situación. Para esta deliberación ya no hay criterios más genéricos, pero sí una cierta orientación comprensiva hacia la vida buena o bienestar.

La primera posibilidad muestra que, a la acción moral, indiferentemente de la fuente de la que provenga, le corresponde siempre un aspecto de segundo nivel. Esta no destaca explícitamente en los casos en los que actuamos moralmente bien, ya que en personas con el carácter ordenado la compasión o uno de los otros motivos del nivel ordinario determinan la acción cuasi por sí mismos. Solo en casos excepcionales en los que estos motivos no son efectivos, por ejemplo, por falta de voluntad o por sobreexigencia de nuestras fuerzas, surte efecto un motivo de segundo nivel al que también se remite Schopenhauer. Según su opinión, "no es del todo necesario que la compasión se estimule en cada caso específico"; más bien la razón generaliza la compasión y la convierte en "un propósito fijo de respetar los derechos de cada uno... de mantenerse libre del autorreproche de ser la causa de sufrimiento ajeno". ${ }^{9}$ El último motivo es seguramente un motivo reflexivo que

9 Schopenhauer 1988, 571. 
se refiere al actor mismo. Así, en contra de la interpretación de Tugendhat, tal motivo se incluye también en la moral de la compasión. De hecho, por razones formales, es aplicable en cada fuente posible de la moral. Pues no poder salir de la estructura de reflexividad pertenece a la constitución de la naturaleza humana. En consecuencia, podemos preguntarnos en lo referente a cada motivo, acción, disposición del carácter: ¿quiero ser una persona que tiene este motivo?

Mi tesis era que esta pregunta no surge en lo concerniente a una aceptación principal de las fuentes de la moral. Surge en situaciones excepcionales del tipo mencionado, y aquí la pregunta no es si queremos ser morales, sino en qué grado queremos ser morales. Quien en su idea de la vida correcta y, con ello, en su autoestima, da más espacio a la moral que lo que exige el mínimo usual de moralidad, necesitará con mayor frecuencia la autoestima como motivo, por lo menos al principio, para compensar el alcance limitado de los afectos en el nivel inferior. Pero si una persona se ha acostumbrado a tal ponderación fuerte de la moral, también los motivos que las fuentes morales comunes proporcionan se vuelven paulatinamente tan condicionados que resultan en una conformidad entre nivel inferior y autoestima.

La compasión, como una de las diferentes fuentes de la moral, se comporta de igual modo que las otras fuentes, y también los así llamados afectos morales, como la indignación, fundamentan solo uno de varios campos de la moral y, por ello, no pueden explicar la moral en conjunto. En lo concerniente al interés por un enfoque moral sustancial ilustrado solo queda preguntar cuál es el contenido de las diferentes normas y virtudes del carácter y hacerlo de tal modo que se disuelvan las limitaciones injustificadas en el alcance de estas fuentes, limitaciones que resultan de prejuicios y no de la naturaleza de los objetos. Y, con ello, podemos regresar a los animales - consecuentemente estos tienen que ser incluidos hasta que su naturaleza lo permita, y cada vez en la forma que resulta del tipo de relación hacia nosotros: como objetos de compasión y cuidado, como un tipo de amigos o como seres vivos independientes cuyos intereses pueden chocar con los nuestros.

Tugendhat supone en el artículo antes mencionado que, posiblemente, acciones inmediatamente altruistas sin auto-referencia ocurren en posturas de la mística. Pero esto, a causa de las explicaciones anteriores, no puede referirse al afecto diario de la compasión, el cual podría fundamentar la moral frente a los animales y el cual se sitúa dentro de las estructuras reflexivas usuales a las que pertenece la moral. Como el mismo Tugendhat supone, el enfoque místico emplea otras estructuras y ya no corresponde a lo que entendemos habitualmente por moral.

De hecho, tampoco Schopenhauer se estanca en la referencia a la compasión como fundamento de la moral, sino que simultáneamente ve en este fenómeno 
"el gran misterio de la ética, su fenómeno originario e hito" sobre el que solo la especulación metafísica todavía puede expresar algo. ${ }^{10}$ Según esto, la posibilidad de identificación se basa en que todas las cosas y seres vivos son objetivaciones de la única voluntad. El individuo humano es tal realización de la voluntad, con la peculiaridad de que tiene conocimientos, o sea, ideas, especialmente también la idea de qué es voluntad o apetencia. Ahora, como objetivación material de la voluntad, el ser humano se encuentra aislado. El individuo aislado sufre, y no solo de vez en cuando, sino constitucionalmente, ya que la voluntad es ilimitada y nunca está satisfecha. La compasión nos advierte de que otros individuos sufren del mismo modo. En cambio, comprender la universalidad del sufrimiento puede llevar a comprender que los límites entre los individuos son arbitrarios. El siguiente paso consiste en comprender que la voluntad es vana y en apartarse de la vida y del querer.

Sin embargo, con ello está claro que el paso a la mística es inapropiado para justificar la consideración de los animales. Más bien, ese paso nos lleva justamente, más allá de la problemática moral, a una actitud de distancia de querer y sufrir. Tal actitud podría ser útil en los casos que consisten en el hecho antes mencionado de que la humanidad ha originado y todavía sigue originando inevitablemente sufrimiento incontable a los animales a través de su expansión y estilo de vida. Pero esa actitud no es razonable en lo que se refiere a problemas como la cría intensiva o los experimentos con animales, cuando el sufrimiento no es inevitable, sino que nosotros podemos cambiar o renunciar a nuestra práctica. El propio Tugendhat notó, después de la lectura del libro Elogio de la Infelicidad de Emilio Lledó, que la conciencia de la miseria provocada por los humanos rompe la tranquilidad mística del alma. ${ }^{11}$ Pero él ha desaprovechado la ocasión de revisar su posición, es decir, de sustituir el desalojamiento de los animales hacia la mística, por cuidar activamente su bienestar dentro de la moral.

(Traducción de Marian Sanclemente Santiago)

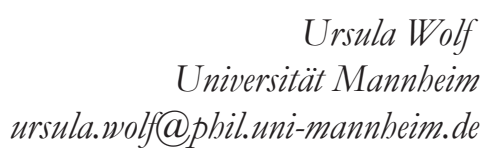

10 Véase mi artículo de 2016 sobre Schopenhauer.

11 Tugendhat 2007, 187ss. 


\section{BIBLIOGRAFÍA}

LLEDÓ, Emilio (2005), Elogio de la Infelicidad, Madrid.

MacIntYre, Alasdair (1999), Dependent Rational Animals, London.

Midgley, Mary (1983), Animals and Why They Matter, Athens (Georgia).

Regan, Tom (1984), The Case for Animal Rights, London.

SCarano, Nico/SuÁreZ, Mauricio, ed. (2005), Ernst Tugendhats Ethik, München.

SChopenhauer, Arthur (1988), Preisschrift über die Grundlage der Moral, Zürich, Werke ed. Lütkehaus, Bd. III.

SCRUTON, Roger (1996), Animal Rights and Wrongs, London.

SEArle, John (1969), Speech Acts, London 1969.

TugENDHAT, Ernst (1993), Vorlesungen über Ethik, Frankfurt a.M.

Tugendhat, Ernst (2005), Das Problem einer aufgeklärten Moral, in Scarano ed., 13-30.

Tugendhat, Ernst (2007), Anthropologie statt Metaphysik, München.

Warren, Mary Anne (1997), Moral Status. Obligations to Persons and Other Living things, Oxford.

Williams, Bernard (1985), Ethics and the Limits of Philosophy, London.

Wolf, Ursula (2005), Für Tiere nur Mitleid? Überlegungen zu den Quellen der Moral, in Scarano, ed., 77-90.

Wolf, Ursula (2012), Ethik der Mensch-Tier-Beziehung, Frankfurt a.M., edición española: Ética de la relación entre humanos y animales, Madrid 2014.

WoLF, Ursula (2016), How Schopenhauer's ethics of compassion can contribute to today's ethical debate, Enrahonar. Quaderns de filosofía 55 (2015), 41-49. 